Carfilzomib (CFZ), an epoxyketone with specific chymotrypsin-like activity, is a second-generation proteasome inhibitor with significant activity in patients with relapsed and refractory multiple myeloma. On July 20, 2012, the US Food and Drug Administration approved CFZ to treat patients with multiple myeloma who have received at least two prior therapies including bortezomib (BORT) and an immunomodulatory agent and have demonstrated disease progression on or within 60 days of completion of the last therapy. Cytogenetic abnormalities did not appear to have a significant impact on the CFZ activity. Carfilzomib was well tolerated and demonstrated promising efficacy in patients with renal insufficiency. Pomalidomide (POM) (CC-4047) is a novel immunomodulatory derivative (IMID) with a stronger in vitro anti-myeloma effect compared with "older" IMIDs thalidomide and lenalidomide (LEN). On February 8, 2013, the US Food and Drug Administration approved POM (Pomalyst, Celgene) for the treatment of MM patients who have received at least two prior therapies including LEN and BORT and have demonstrated progression on or within 60 days of completion of the last therapy. Pomalidomide is a novel IMID with significant anti-myeloma activity and manageable toxicity. This compound has shown high efficacy in MM patients who were resistant to prior use of LEN/BORT as well as in patients with a high-risk cytogenetic profile. Carfilzomib and POM have very high efficacy and will be used also in first line therapy in future.

Key words: multiple myeloma, treatment, carfilzomib, pomalidomide.

Contemp Oncol (Pozn) 2014; 18 (1): 17-21 DOI: $10.5114 /$ wo.2014.40175

\section{New drugs in multiple myeloma - role of carfilzomib and pomalidomide}

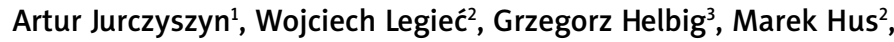 \\ Sławomira Kyrcz-Krzemieńn ${ }^{3}$, Aleksander B. Skotnicki ${ }^{4}$
}

1'Department of Hematology, Krakow University Hospital, Poland

2Department of Hematooncology and Bone Marrow Transplantation, Medical University of Lublin, Poland

${ }^{3}$ Department of Hematology and Bone Marrow Transplantation, Silesian Medical University, Katowice, Poland

${ }^{4}$ Chair and Department of Hematology, Jagiellonian University Medical College, Krakow, Poland

Carfilzomib (CFZ), an epoxyketone with specific chymotrypsin-like activity, is a second-generation proteasome inhibitor with significant activity in patients with relapsed and refractory multiple myeloma [1]. Carfilzomib selectively inhibits activity of a chymotrypsin-like ( $\beta 5$ ) subunit of the 205 proteasome and has minimal cross-reactivity with other protease classes. This inhibition is irreversible, as well as dose- and time-dependent in vitro and in vivo. As a result of proteasome inhibition we observe the accumulation of polyubiquitinated proteins and induction of apoptosis in many tumor cell lines including not only multiple myeloma but also Waldenström macroglobulinemia, acute myeloid leukemia, B-cell malignancies (Burkitt's and NHL), pancreatic cancer, and lung carcinoma $[2,3]$.

The efficacy of CFZ in heavily pretreated, relapsed and refractory multiple myeloma has been evaluated in a number of phase II trials. The pivotal PX171-003-A1 study of single-agent CFZ enrolled 266 patients [1]. The median age was 63 years and the patients had received a median of 5 prior therapies including lenalidomide, bortezomib, thalidomide, alkylating agents, steroids, anthracyclines and transplants. Carfilzomib was administered intravenously (for 3 weeks in each 4-week cycle), twice weekly, at a daily dose of $20 \mathrm{mg} / \mathrm{m}^{2}$ in cycle 1 and then at a dose of $27 \mathrm{mg} / \mathrm{m}^{2}$ twice weekly for up to 12 cycles. The ORR was $23.7 \%$, the clinical benefit response (CBR) was $37 \%$, the median PFS was 3.7 months and the median duration of response (DOR) was 7.8 months $[C B R=O R R(S C R+C R+V G P R+P R)+M R$ (minimal response)]. The median $O S$ in the group of patients refractory to both bortezomib and lenalidomide was 11.9 months. Cytogenetic abnormalities did not appear to have a significant impact on CFZ activity. The ORR in patients with unfavorable cytogenetic or FISH findings was higher (29.6\%) compared with patients with normal-favorable cytogenetics/FISH (22.8\%), although the newest data revealed similar ORR in both groups but significantly higher OR in the standard risk group [4].

The study PX-171-004 with single-agent CFZ evaluated two groups of patients: the first bortezomib-naive and the second previously treated with bortezomib. In the bortezomib-naive cohort the ORR was $47.6 \%$ and CBR was $61.9 \%$ after six cycles of therapy [5]. In the bortezomib-treated cohort the ORR was $17.1 \%$ and CBR was $31.4 \%$. The median DOR was $>10.6$ months, while the median time to progression (TTP) and median PFS were 5.3 months [6].

Patients with renal insufficiency were evaluated in the PX-171-005 study. Carfilzomib was administered at a dose of $15 \mathrm{mg} / \mathrm{m}^{2}$ in cycle 1 , then was 
escalated to $20 \mathrm{mg} / \mathrm{m}^{2}$ in cycle 2 and to $27 \mathrm{mg} / \mathrm{m}^{2}$ from cycle 3 up to 12 cycles [7]. A similar assessment was summarized in the study by Badros et al. [8]. The pharmacokinetics and safety of carfilzomib were not influenced by the degree of baseline renal impairment, including in patients on dialysis, and carfilzomib was well tolerated and demonstrated promising efficacy [7, 8].

Carfilzomib has an acceptable tolerability profile. The incidence of grade 3 and 4 adverse events is low [9]. In the pivotal PX-171-003-A1 trial, grade 3 or 4 thrombocytopenia and anemia were observed in 29 and $24 \%$ of patients respectively. They were not dose limiting and did not result in treatment discontinuation. In the PX-171-005 study the incidence of adverse events was independent of renal status [7].

Recommended dosage of intravenous CFZ in relapsed, or relapsed and refractory, multiple myeloma is $20 \mathrm{mg} / \mathrm{m}^{2}$ on days 1, 2, 8, 9, 15 and 16 of the 28-day treatment cycle. If the therapy is well tolerated in cycle 1 , the dose should be increased to $27 \mathrm{mg} / \mathrm{m}^{2}$ in subsequent cycles. The maximum BSA to be used for dose calculation is $2.2 \mathrm{~m}^{2}$. Therapy with CFZ should be continued until disease progression or unacceptable toxicity occurs [10].

On July 20, 2012, the US Food and Drug Administration approved CFZ to treat patients with multiple myeloma who have received at least two prior therapies including bortezomib and an immunomodulatory agent and have demonstrated disease progression on or within 60 days of completion of the last therapy.

Several trials of CFZ were presented at the 2012 ASH meeting. There were the combination of CFZ with thalidomide and dexamethasone reported by Sonneveld et al. [11], and thalidomide, dexamethasone and cyclophosphamide reported by Mikhael et al. [12]. Palumbo et al. [13] presented the results of CCd (carfilzomib, cyclophosphamide and dexamethasone) in newly diagnosed, elderly MM patients. Korde et al. [14] presented a phase II clinical and correlative study of carfilzomib, lenalidomide, and dexamethasone (CRd) in newly diagnosed MM patients. Alsina et al. [15] presented a poster with updated results of a phase II study of cyclophosphamide, bortezomib, pegylated doxorubicin, and dexamethasone (CVDD), in patients with newly diagnosed myeloma.

All these drug combinations have shown their effectiveness in resistant, refractory MM patients. The results are encouraging, and need confirmation on larger cohorts of patients.

In conclusion, CFZ is a second-generation proteasome inhibitor with substantial activity in heavily pretreated multiple myeloma patients. Carfilzomib is well tolerated with a low number of patients discontinuing therapy due to adverse events. No peripheral neuropathy was connected with CFZ, even in patients with baseline symptoms. Carfilzomib was well tolerated and demonstrated promising efficacy in patient with renal insufficiency.

We are also waiting for results of ongoing trials assessing CFZ [ENDEAVOR Study: A Randomized, Open-Label, Phase 3 Study of Carfilzomib Plus Dexamethasone vs. Bortezomib Plus Dexamethasone in Patients with Relapsed Multiple Myeloma. FOCUS Study: A randomised,
Open-label, Phase 3 Study of Carfilzomib vs. Best Supportive Care in Subjects with Relapsed and Refractory Multiple Myeloma. ASPIRE Study: A randomised, Multicenter, Phase 3 Study Comparing Carfilzomib, Lenalidomide, and dexamethasone (Crd) vs. Lenalidomide and Dexamethasone (Rd) in Subjects with Relapsed Multiple Myeloma], which may supply us with a fresh look at CFZ in multiple myeloma.

Another new drug in therapy of multiple myeloma is pomalidomide (CC-4047) - a novel immunomodulatory derivative (IMID) with a stronger in vitro anti-myeloma effect compared with "older" IMIDs - thalidomide and lenalidomide. IMIDs are found to be highly effective in myeloma patients and their anti-cancer activity includes several mechanisms. Namely, they may induce apoptosis via caspase-8, inhibit angiogenesis and cytokine secretion, and impede the interactions between stroma and myeloma cells [16]. Finally, the protein cereblon (CRBN) has been recently identified as a target for IMIDs. Low CRBN expression was found to be associated with IMID resistance in myeloma cell lines. It was demonstrated that CRBN targets interferon regulatory factor 4 , which plays a crucial role in myeloma cell survival. Moreover, it down-regulates tumor necrosis factor- $\alpha$ [17]. It has also been observed that decreased CRBN mRNA expression correlated with shorter progression-free survival and overall survival in patients with refractory and relapsed multiple myeloma (MM) [18].

The efficacy, safety and immunomodulatory effects of pomalidomide (POM) as a single agent in relapsed/refractory $M M$ have been explored in two phase I studies. A total of $24 \mathrm{MM}$ patients were included in the study of Schey et al. [19]. The maximum tolerated dose was established to be $2 \mathrm{mg}$ daily. Nineteen out of the 24 patients continued the study beyond four weeks. It was demonstrated that despite a median of three prior lines of therapy including thalidomide and autologous hematopoietic stem cell transplantation (AHSCT), 67\% of the patients achieved at least $25 \%$ reduction in monoclonal protein concentration, 54\% experienced a greater than $50 \%$ decrease, and in 4 out of the 24 patients (17\%) the criteria for complete response (CR) were met. Moreover, in vivo T-cell costimulation of this compound was also noted. Namely, it significantly increased serum interleukin (IL)-2 receptor and IL-12 levels. It should be emphasized that POM was well tolerated and no serious non-hematologic adverse events were observed. However, regarding the safety concerns, there was a single report demonstrating a higher risk of deep vein thrombosis in MM patients on POM [20]. The same group reported on the results of a phase I study with alternate day POM. They included 20 heavily pretreated patients and $50 \%$ of them achieved a greater than $50 \%$ decrease in paraprotein. $10 \%$ of included patients met criteria for CR. The authors concluded that alternate day POM retained strong anti-myeloma activity whilst decreasing the incidence of thromboembolism [21]. However, it should be noted that these studies were performed in a pre-bortezomib and pre-lenalidomide era. Recently, the results of a phase I study of POM in MM patients treated previously with bortezomib 
(BORT) and lenalidomide (LEN) have been published. Oral POM was administered on days 1-21 of each 28-day cycle. Thirty-eight patients who had received a median of 6 prior therapies with BORT and LEN were included in this study. Dexamethasone (DEX) at 40 mg daily was allowed from the $4^{\text {th }}$ cycle for those who progressed or had achieved less than a minimal response. The maximum tolerated dose of POM was established to be $4 \mathrm{mg}$ for a 28-day cycle. Finally, DEX was added to POM in $22 \mathrm{pa-}$ tients. $21 \%$ of the patients achieved $>50 \%$ decrease of paraprotein and 3\% fulfilled the CR criteria. The toxicity was manageable [22].

The first phase II study of POM in combination with low-dose DEX (POM/DEX) in relapsed MM enrolled $60 \mathrm{pa}$ tients. Pomalidomide was administered orally at $2 \mathrm{mg}$ daily on days 1-21 of a 28-day cycle, and DEX at $40 \mathrm{mg}$ daily weekly. $63 \%$ of evaluated patients responded. Twenty patients (33\%) achieved greater than very good partial response (VGPR), including 3 who met criteria for CR. The efficacy of this combination was also seen in patients previously refractory to thalidomide (37\%), LEN $(40 \%)$ and BORT (60\%) treatment. Moreover, $74 \%$ of the patients with a high-risk cytogenetic profile responded to the therapy. Myelosuppression was the most common toxicity [23]. The Mayo Group reported the results of $\mathrm{POM} / \mathrm{DEX}$ combination in MM patients refractory to LEN. Thirty-four patients were included in this study and the overall response rate (ORR) was 47\% including 11 cases with at least a partial response (PR). The median overall survival (OS) was 13.9 months [24]. The same group compared the efficacy of two different doses of POM (2 mg and $4 \mathrm{mg}$ ) in patients with dual refractoriness to LEN and BORT. DEX at $40 \mathrm{mg}$ was administered weekly. A total of 70 patients (35 in each cohort) were enrolled. No response to treatment in the 2-mg group was observed in $49 \%$ of patients whereas ORR was $43 \%$ for the 4 -mg cohort. It should be emphasized that responses in both cohorts were rapid and durable, but the follow-up was too short to draw final conclusions. Overall survival at 6 months was estimated to be $78 \%$ and $67 \%$ for $2 \mathrm{mg}$ and $4 \mathrm{mg}$ groups respectively. Nevertheless, the results of this trial were highly encouraging if we consider the studied population and that there was no dose-response effect. However, one should keep in mind the frequent occurrence of severe myelosuppression, especially neutropenia and thrombocytopenia, observed in this study. It may be partially explained by refractoriness of the disease and prior treatment. The risk of thrombotic complications remained low while on low-dose DEX and adequate prophylaxis. $11 \%$ of the patients had peripheral neuropathy of grade 2 in both dose cohorts [25]. The follow-up results of the above-mentioned study based on $345 \mathrm{MM}$ patients were presented during the ASH Meeting in 2012. At least PR was seen in $34 \%$ of the patients [26]. Recently, the results of two different dose regimens of POM/DEX in LEN and BORT refractory MM have been presented by the French Myeloma Group. Eighty-four patients with a median of 5 prior lines of therapy were included in this randomized study. Pomalidomide at $4 \mathrm{mg}$ daily was administered continuously for 28 days over a 28-day cycle or for 21 days of a 28-day cycle. ORR was similar between groups: $34 \%$ and $35 \%$ respectively. After the median follow-up of 23 months, the median survival was 14.9 months with 44\% of patients alive [27]. Finally, the largest multicenter randomized phase III study to date comparing POM/DEX versus high-dose DEX alone was recently initiated. Four hundred and fifty-five patients with dual refractoriness (LEN and BORT or intolerant to BORT only) were enrolled in this study. Progression-free survival was significantly longer in the POM/DEX group compared with DEX alone (15.7 weeks vs. 8.0 weeks). A benefit regarding overall survival was also noted for this former subgroup (median not reached vs. 34 weeks) [28].

Combinations of POM with other agents were also tested in patients with refractory and relapsed MM. Palumbo et al. presented the results of a phase I/II study of POM combined with cyclophosphamide and prednisone (PCP) in LEN refractory/relapsed patients. At least PR was documented in $73 \%$ of the patients resistant to LEN [29]. An overall response rate of $60 \%$ (including $27 \%$ meeting the criteria for very good partial response) in refractory MM patients was achieved with the combination of POM/DEX and clarithromycin [30].

The toxicity of POM/DEX combinations in heavily pretreated MM patients is acceptable. Neutropenia grade $3 / 4$ remained the major hematologic toxicity [26]. Among non-hematologic adverse events, fatigue was the most common complaint [25]. The frequency of thrombotic complications was low; they occurred in about 3\% of patients [26]. A small proportion of patients may suffer from peripheral neuropathy [25] or noninfectious pulmonary injury [31].

On February 8, 2013, the US Food and Drug Administration approved POM (Pomalyst, Celgene) for the treatment of MM patients who have received at least two prior therapies including LEN and BORT and have demonstrated progression on or within 60 days of completion of the last therapy. This approval was based on the results of a phase II study with a total of 221 patients resistant to LEN/BORT. Patients were randomized to receive $4 \mathrm{mg}$ daily POM alone for 21 days of a 28 -day cycle vs. POM/DEX. At least PR was achieved in $15 \%$ and $34 \%$ of patients respectively [32].

In conclusion, POM is a novel IMID with significant anti-myeloma activity and manageable toxicity. This compound has shown high efficacy in MM patients who were resistant to prior use of LEN/BORT as well as in patients with a high-risk cytogenetic profile.

Finally, although this article focuses primarily on these agents in the relapse setting, it should be noted that once evaluated in the relapse setting, these agents will ultimately be evaluated in newly diagnosed patients. This is the case with an ongoing phase 3 clinical trial of elotuzumab plus lenalidomide and dexamethasone, compared to lenalidomide and dexamethasone alone, in the frontline treatment of myeloma. Also, once approved, many of these agents have increased efficacy when combined with different class drugs. For example, CRF, lenalidomide and dexamethasone is a very potent regimen for relapse and frontline therapy. 
In summary, the future pipeline for myeloma agents is very promising. Although myeloma represents only $1 \%$ of all cancers, six of the 21 agents approved over the past 12 years to treat cancer have been for myeloma, and more myeloma agents appear on the horizon. Soon, we truly will be able to state with confidence that myeloma is a chronic disease with multiple treatment options that can control the disease for years.

\section{Authors declare no conflict of interest.}

\section{References}

1. Siegel DS, Martin T, Wang M, et al. A phase 2 study of single-agent carfilzomib (PX-171-003-A1) in patients with relapsed and refractory multiple myeloma. Blood 2012: 120: 2817-25.

2. Dimopoulos MA, Terpos E, Kastritis E. Proteasome inhibitor therapy for Waldenström's macroglobulinemia. Clin Lymphoma Myeloma Leuk 2013; 13: 235-7.

3. Demo SD, Kirk CJ, Aujay MA, et al. Antitumor activity of PR-171, a novel irreversible inhibitor of the proteasome. Cancer Res 2007; 67: 6383-6391.

4. Jakubowiak AJ, Siegel DS, Martin T, et al. Treatment outcomes in patients with relapsed and refractory multiple myeloma and highrisk cytogenetics receiving single-agent carfilzomib in the PX-171003-A1 study. Leukemia 2013; 27: 2351-6.

5. Vij R, Wang M, Kaufman JL, et al. An open-label, single-arm, phase 2 (PX-171-004) study of single-agent carfilzomib in bortezomib-naive patients with relapsed and/or refractory multiple myeloma. Blood 2012; 119: 5661-70.

6. Vij R, Siegel DS, Jagannath S, et al. An open-label, single-arm, phase 2 study of single-agent carfilzomib in patients with relapsed and/ or refractory multiple myeloma who have been previously treated with bortezomib. Br J Hematol 2012; 158: 739-48.

7. Niesvizky R, Vij R, Martin T, et al. Carfilzomib pharmacokinetics, safety and activity in patients with relapsed or refractory multiple myeloma and renal dysfunction: final results (abstract no. 0890). Haematologica 2011; 96 Suppl. 2: 370-1.

8. Badros AZ, Vij R, Martin T, Zonder JA, Kunkel L, Wang Z, Lee S, Wong AF, Niesvizky R. Carfilzomib in multiple myeloma patients with renal impairment: pharmacokinetics and safety. Leukemia 2013; 27: 1707-14

9. US FDA. NDA 202,714, Onyx Pharmaceuticals, Inc., Proposed Trade Name: Kyprolis (Carfilzomib for Injection), Briefing Document, ODAC Meeting Date: 20 June 2012, Submission Date: 17 May 2012, Available from URL: http://www.fda.gov/downloads/AdvisoryCommittees/CommitteesMeetingMaterials/Drugs/OncologicDrugs AdvisoryCommittee/UCM308565.pdf.

10. US FDA. KYPROLISTM (carfilzomib) for Injection, for intravenous use Initial U.S. Approval: 2012. Prescription information. Available from URL: http://www.accessdata.fda.gov/drugsatfda_docs/ label/2012/202714lbl.pdf.

11. Sonneveld P, Asselbergs E, Zweegman S, et al. Carfilzomib combined with thalidomide and dexamethasone (CTD) is a highly effective induction and consolidation treatment in newly diagnosed patients with multiple myeloma $(\mathrm{mm})$ who are transplant candidates. Paper presented at: 54th American Society of Hematology Annual Meeting and Exposition; December 8-11, 2012; Atlanta, GA.

12. Mikhael JR, Reeder CB, Libby EN III, et al. Results from the phase Il dose expansion of cyclophosphamide, carfilzomib, thalidomide and dexamethasone (CYCLONE) in patients with newly diagnosed multiple myeloma. Paper presented at: $54^{\text {th }}$ American Society of Hematology Annual Meeting and Exposition; December 8-11, 2012; Atlanta, GA.

13. Palumbo A, Bringhen S, Villani O, et al. Carfilzomib, cyclophosphamide and dexamethasone (CCd) for newly diagnosed multiple myeloma (MM) patients. Paper presented at: $54^{\text {th }}$ American Society of Hematology Annual Meeting and Exposition; December 8-11, 2012; Atlanta, GA.

14. Korde N, Zingone A, Kwok M, et al. Phase II clinical and correlative study of carfilzomib, lenalidomide, and dexamethasone (CRd) in newly diagnosed multiple myeloma (MM) patients. Paper presented at: $54^{\text {th }}$ American Society of Hematology Annual Meeting and Exposition; December 8-11, 2012; Atlanta, GA.

15. Alsina M, Baz R, Shain KH, et al. Updated results of phase II study of cyclophosphamide (Cy), bortezomib (Bz), pegylated doxorubicin (DOX), and dexamethasone (dex),(CVDD), in patients with newly diagnosed myeloma: an effective induction regimen for high risk disease. Poster presented at: $54^{\text {th }}$ American Society of Hematology Annual Meeting and Exposition; December 8-11, 2012; Atlanta, GA.

16. Cavo M. A third-generation IMID for MM. Blood 2011; 118: 2931-2.

17. Zhu YX, Kortuem KM, Stewart AK. Molecular mechanism of action of immune-modulatory drugs thalidomide, lenalidomide and pomalidomide in multiple myeloma. Leuk Lymphoma 2013; 54: 683-7.

18. Schuster S, Kortuem K, Zhu Y, et al. Cereblon expression predicts response, progression free and overall survival after pomalidomide and dexamethasone therapy in multiple myeloma. Blood (American Society of Hematology Annual Meeting) 2012; 120: abstract 194.

19. Schey SA, Fields P, Bartlett JB, et al. Phase I study of an immunomodulatory thalidomide analog, CC-4047, in relapsed or refractory multiple myeloma. J Clin Oncol 2004; 22: 3269-76.

20. Streetly M, Hunt BJ, Parmar K, et al. Markers of endothelial and haemostatic function in the treatment of relapsed myeloma with the immunomodulatory agent Actimid (CC-4047) and their relationship with venous thrombosis. Eur J Haematol 2005; 74: 293-6.

21. Streetly MJ, Gyertson K, Daniel Y, et al. Alternate day pomalidomide retains anti-myeloma effect with reduced adverse events and evidence of in vivo immunomodulation. Br J Haematol 2008; 141: 41-51.

22. Richardson PG, Siegel D, Baz R, et al. Phase 1 study of pomalidomide MTD, safety, and efficacy in patients with refractory multiple myeloma who have received lenbalidomide and bortezomib. Blood 2013; 121: 1961-7.

23. Lacy MQ, Hayman SR, Gertz MA, et al. Pomalidomide (CC4047) plus low-dose dexamethasone as therapy for relapsed multiple myeloma. J Clin Oncol 2009; 27: 5008-14.

24. Lacy MQ, Haymann SR, Gertz MA, et al. Pomalidomide (CC4047) plus low dose dexamethasone (Pom/dex) is active and well tolerated in lenalidomide refractory multiple myeloma (MM). Leukemia 2010; 24: 1934-9.

25. Lacy MO, Allred JB, Gertz MA, et al. Pomalidomide plus low-dose dexamethasone in myeloma refractory to both bortezomib and lenalidomde: comparison of 2 dosing strategies In dual-refractory disease. Blood 2011; 118: 2970-5.

26. Lacy MQ, Kumar SK, LaPlant BR, et al. Pomalidomide plus low dose dexamethasone (Pom/Dex) in relapsed myeloma: long term follow up and factors predicting outcome in 345 patients. Blood (American Society of Hematology Annual Meeting) 2012; 120: abstract 201.

27. Leleu X, Attal M, Arnulf B, et al. Pomalidomide plus low-dose dexamethasone is active and well tolerated in bortezomib and lenalidomide-refractory multiple myeloma: Intergroupe Francophone du Myelome 2009-02. Blood 2013; 121: 1968-75.

28. Dimopoulos MA, Lacy MO, Moreau P, et al. Pomalidomide in combination with lowOdose dexamethasone: demonstrates a significant progression free survival and overall survival advantage, in relapsed/refractory MM: a phase 3, multicenter, randomized, open-label study. Blood (American Society of Hematology Annual Meeting) 2012; 120: abstract 6.

29. Palumbo A, Larocca A, Carella AM, et al. A phase I/II study of pomalidomide-cyclophosphamide-prednisone (PCP) in patients with multiple myeloma relapsed/refractory to lenalidomide. Blood (American Society of Hematology Annual Meeting) 2012; 120: abstract 446.

30. Tomer MM, Rodriguez M, Shah M, et al. ClaPD (clarithromycin/ [Biaxin], pomalidomide, dexamethasone) therapy in relapsed or 
refractory multiple myeloma. Blood (American Society of Hematology Annual Meeting) 2012; 120: abstract 77.

31. Geyer HL, Viggiano RW, Lacy MQ, et al. Acute lung toxicity related to pomalidomide. Chest 2011; 140: 529-33.

32. Jagannath S, Hofmeister CC, Siegel DS, et al. Pomalidomide (Pom) with low-dose dexamethasone (LoDex) in patients with relapsed and refractory multiple myeloma who have received prior therapy with lenalidomide and bortezomib: updated phase 2 results and age subgroup analysis. Blood (American Society of Hematology Annual Meeting) 2012; 120: abstract 450.

\section{Address for correspondence}

Jurczyszyn Artur MD, PhD

Klinika Hematologii, Szpital Uniwersytecki

Kopernika 17

31-501 Krakow, Poland

tel. +48 601539077

e-mail: mmjurczy@cyf-kr.edu.pl

Submitted: 4.06.2013

Accepted: $\quad 16.10 .2013$ 